\title{
Endoscopic submucosal dissection and tunneling procedures using a novel all-in-one bipolar device $\square$
}

\section{(ㄷ)(1) $\odot$}

Authors

Zaheer Nabi ${ }^{1}$, Radhika Chavan ${ }^{2}$, Mohan Ramchandani ${ }^{2}$, Zacharias Tsiamoulos ${ }^{3}$, Jahanqeer Basha ${ }^{4}$, Rajesh Goud $^{2}$, Santosh Darisetty ${ }^{2,5}$, Nageshwar Reddy ${ }^{2}$

Institutions

1 Asian Institute of Gastroenterology - Medical Gastroenterology, Hyderabad, India

2 Asian Institute of Gastroenterology - Gastroenterology, Hyderabad, India

3 St. Mark's Hospital - Gastroenterology, Harrow, UK

4 PGIMER - Gastroenterology, Chandigarh, India

5 Asian Institute of Gastroenterology - Anesthesia, Hyderabad, India

submitted 9.4.2020

accepted after revision 22.5.2020

\section{Bibliography}

Endoscopy International Open 2020; 08: E1302-E1307

DOI 10.1055/a-1220-6562

ISSN 2364-3722

(C) 2020. The Author(s).

This is an open access article published by Thieme under the terms of the Creative Commons Attribution-NonDerivative-NonCommercial License, permitting copying and reproduction so long as the original work is given appropriate credit. Contents may not be used for commecial purposes, or adapted, remixed, transformed or built upon. (https://creativecommons.org/licenses/by-nc-nd/4.0/)

Corresponding author

Zaheer Nabi, Asian Institute of Gastroenterology -

Gastroenterology, Asian Institute of Gastroenterology,

Hyderabad 500082, India

Fax: + 91-40-23324255

zaheernabi1978@gmail.com

\section{ABSTRACT}

Background and study aims Recent innovations in devices and techniques have revolutionized the field of endoscopic resection procedures. In this study, we evaluated the safety and feasibility of endoscopic submucosal dissection and tunneling procedures with a novel, multipurpose bipolar device.

Patients and methods Data from consecutive patients who underwent per-oral endoscopic myotomy (POEM), submucosal tunneling endoscopic resection (STER), and endoscopic submucosal dissection (ESD) using a novel bipolar device (December 2019 to February 2020) were analyzed retrospectively. Procedure duration, technical success, and adverse events (AEs) were recorded.

Results A total of 10 procedures were performed using the novel bipolar device during the study period. The procedures included POEM $(n=7)$, STER for esophageal sub-epithelial tumor $(n=1)$, ESD for rectal polyp $(n=1)$, and gastric neuroendocrine tumor $(n=1)$. POEM was successfully completed in all patients. In patients who underwent STER and ESD, en-bloc resection was achieved in all. Mean procedure time for submucosal tunneling procedures (POEM and STER) was $59.12 \pm 31.12$ minutes. The procedures were completed without the requirement for exchange of accessories in eight cases ( $80 \%)$. There were no major AEs. Mild and moderate AEs occurred during POEM and included capno-peritoneum $(n=1)$, retroperitoneal $\mathrm{CO}_{2}(n=1)$, and empyema $(n=1)$.

Conclusion ESD and tunneling procedures can be safely performed without the need for exchange of accessories using a novel bipolar device. Randomized comparison with conventional knives isrequired to confirm the utility of this device.

\section{Introduction}

Endoscopic submucosal dissection (ESD) and per-oral endoscopic myotomy (POEM) are established therapeutic procedures for management of early gastrointestinal neoplasms and achalasia cardia, respectively [1] Use of several different types of knives has been reported for performing these procedures [2-5]. Recent innovations involving the technique as well as the devices used for tissue dissection have reduced the com- plexity associated with these advanced endoscopic procedures. In this regard, incorporation of water jet facility in newer electrosurgical knives has allowed endoscopists to accomplish the procedure with less need to change the accessories [6 - 8]. However, exchange of accessories is still required for initial submucosal lifting injection and coagulation of larger vessels. In addition, there is a risk of collateral thermal injury due to the monopolar nature of the currently available electrosurgical knives. 
In this series, we evaluated use of a new multifunctional bipolar device for performing POEM, submucosal tunneling endoscopic resection (STER) and endoscopic submucosal dissection (ESD).

\section{Patients and methods}

Data from all patients who underwent endoscopic submucosal tunneling or dissection procedures using the new device from December 2019 to February 2020 were analyzed, retrospectively. Endoscopic procedures included POEM for idiopathic non-sigmoid achalasia, STER and ESD. Exclusion criteria were contraindication to general anesthesia, sigmoid esophagus, failure to provide informed consent, children $<18$ years, predominantly exophytic submucosal lesion, and suspected deep submucosal invasion on narrow-band imaging (NBI). All the procedures were performed by endoscopists (ZA and MR) with adequate experience in POEM, STER, and ESD.

The study was approved by the institution's review board committee and informed consent was obtained from all the patients.

\section{Pre-procedure evaluation}

All patients underwent standard pre-anesthetic assessment. Subtyping of achalasia was performed using high-resolution esophageal topography. Submucosal lesions were assessed with endosonography and cross-sectional imaging. NBI was performed for laterally spreading lesions in the rectum to determine the depth of invasion and suitability for endoscopic resection.

\section{Device and accessories}

Therapeutic channel (3.7 mm) endoscope (GIF-1TH190; Olympus, Tokyo, Japan), tapered transparent cap (DH-28GR; Fujifilm, Tokyo, Japan), bipolar device (Speedboat-RS2; Creo Medical Ltd, Chepstow, Wales, UK), endoscopic clips (EZ Clip, HX610 -090L; Olympus Corp.), and coagulation forceps (Coagrasper G, FD-412LR, Olympus, Japan) were used.

\section{Novel bipolar device}

In this case series, we used a novel bipolar device (SpeedboatRS2; Creo Medical Ltd, Chepstow, Wales, UK) for all the procedures ( $\triangleright$ Fig. 1a and $\triangleright$ Fig. 1b). This multimodality device comprises a boat shaped blade with front and lateral cutting edges, an insulated upper surface, and a protective hull at the bottom ( $\triangleright$ Fig. 1b). The device is fully rotatable, has a working length of $2300 \mathrm{~mm}$, and requires a working channel diameter of at least $3.7 \mathrm{~mm}$. Integrated injection needle $(26 \mathrm{G})$ is incorporated within the device, allowing submucosal injection and irrigation. The device can be rotated with the help of the assistant for performing mucosal lifting injection, mucosal incision, and submucosal dissection ( $\triangleright$ Fig. 2a, $\triangleright$ Fig. 2b, $\triangleright$ Fig. 2c).

This bipolar device uses radiofrequency energy for cutting (400 kHz and $35 \mathrm{~W}$ ) and microwave for coagulation (frequency $5.8 \mathrm{GHz}$, power setting $10 \mathrm{~W}$ ). A designated electrosurgical generator is required while using this device (Creo Medical electrosurgery generator 7-EMR-050)( Fig. 1a).

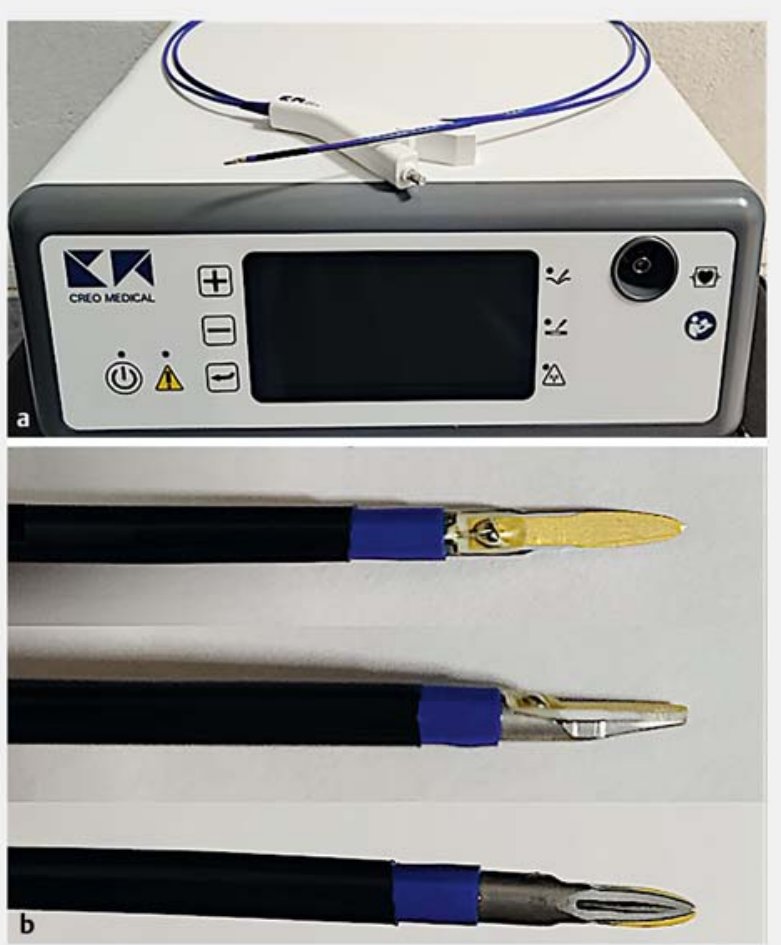

- Fig. 1 Novel bipolar radiofrequency device. a Electrosurgical generator compatible with the bipolar knife. b Endoscopic view demonstrating the novel device in different orientations.

\section{Technique of POEM, STER and ESD}

We have previously described the techniques of POEM and STER using this novel bipolar device $[9,10]$. In all the cases, the entire procedure including incision, dissection, and coagulation was accomplished using the same device. POEM and STER were performed under general anesthesia with the patients in supine position. Whereas, ESD procedures were performed under propofol sedation with patients in left lateral position. A single dose of prophylactic antibiotics (Piperacillin-Tazobactum $4.5 \mathrm{~g}$ ) was administered 30 minutes prior to the tunneling procedures.

In brief, the technique of POEM using the new device is as follows. The device was inserted through the biopsy channel of the therapeutic endoscope. Subsequently, it was rotated so that the exit of the integrated needle from the undersurface of the device could be visualized endoscopically. Submucosal injection using diluted indigocarmine dye was given to create an adequate mucosal bulge ( $\triangleright$ Fig. 3a). The device was rotated so that the lateral cutting edges were oriented perpendicular to the esophageal mucosa. Mucosal incision was performed by gently pressing the device over the bulge created in the previous step ( $\triangleright$ Fig.3b). Submucosal tunneling was performed with the device in the neutral position i.e. protective hull at the bottom, curved cutting edges on the lateral sides, and golden yellow insulated surface on the top ( $>$ Fig. $3 \mathbf{c}$ ). Dissection of the submucosal fibers was performed in a left to right or vice versa direction. 

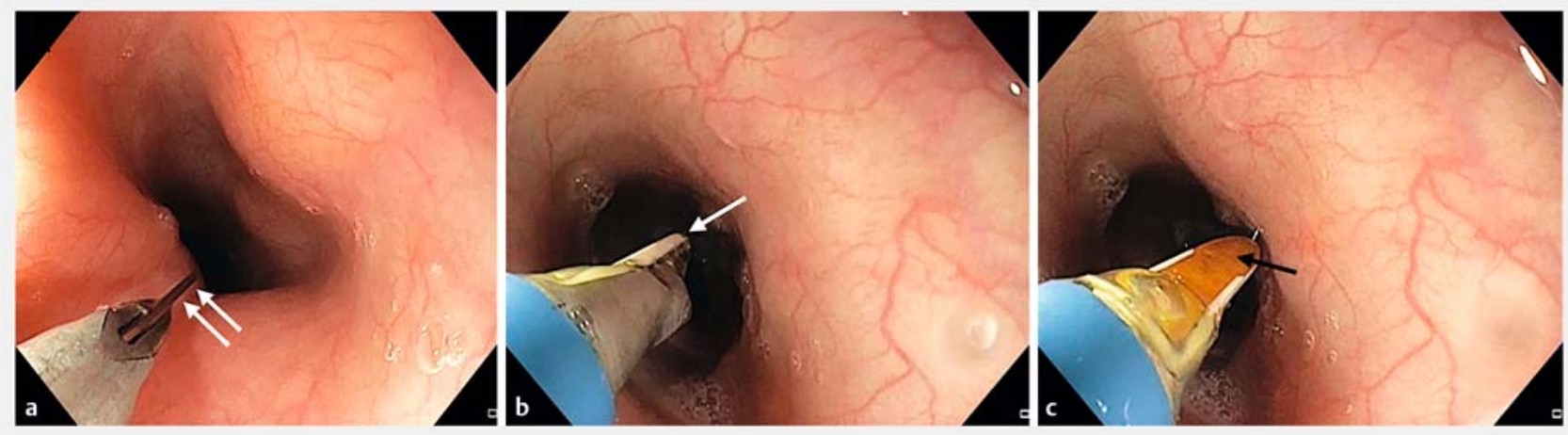

- Fig. 2 Endoscopic images depicting the orientation of the device for different steps during the POEM procedure. a Orientation of the knife for performing initial mucosal lifting injection (Note the needle exiting from the undersurface of the knife). $\mathbf{b}$ Orientation of the knife for performing mucosal incision and myotomy during POEM (Note the position of lateral cutting edges relative to the esophageal mucosa). c Orientation of the knife for performing submucosal tunneling during POEM (Note that the lateral cutting edges can be used to cut on either side i. e. left or right).
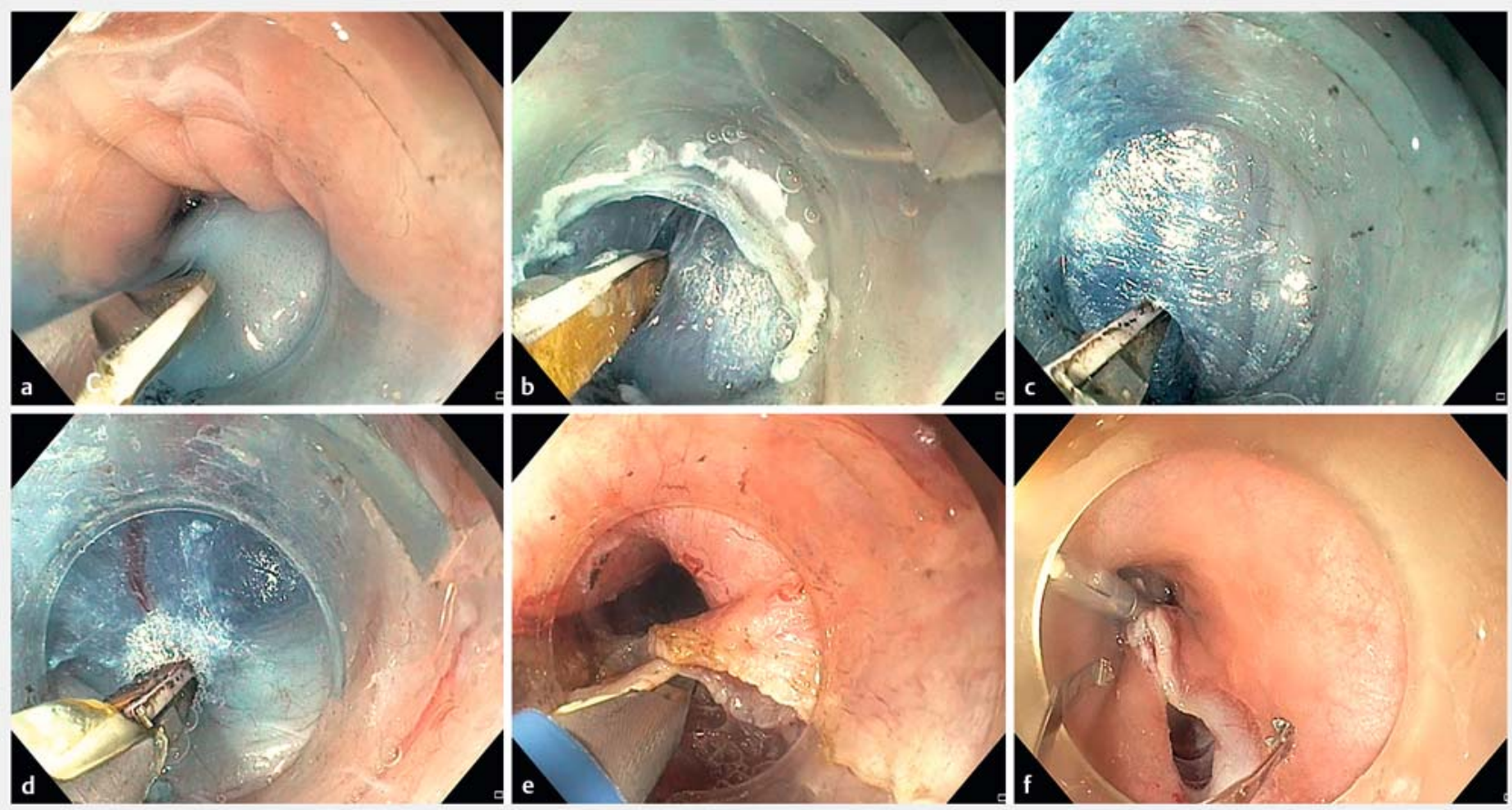

Fig. 3 Per-oral endoscopic myotomy using the new bipolar device. a Submucosal injection using saline mixed with indigo carmine. b Mucosal incision after rotating the device so that the lateral cutting edge comes in contact with the mucosa. c Submucosal tunneling with the same device (note orientation of device). $\mathbf{d}$ Coagulation of vessels using microwave energy. e Myotomy (circular only in the upper part and full thickness in the lower part). $\mathbf{f}$ Closure of mucosal incision with multiple endoclips.

Radiofrequency energy with similar settings $(400 \mathrm{kHz}$ and $35 \mathrm{~W}$ ) was used for mucosal incision, submucosal dissection, and myotomy. Coagulation of intervening vessels was performed using the same device using microwave energy (frequency $5.8 \mathrm{GHz}$, power setting $10 \mathrm{~W}$ ) ( $\triangleright \mathbf{F i g} . \mathbf{3} \mathbf{d})$. The tip or the curved blade near the tip was gently pressed onto the bleeding site and coagulation current applied for 5 to 10 seconds. The appearance of small bubbles indicated ongoing coagulation and adequate contact of the device with the bleeding surface.
Myotomy was performed after rotating the knife in an orientation similar to the one used for mucosal incision ( $\vee$ Fig. 3e). Finally, the mucosal incision was closed using endoclips ( $\triangleright$ Fig. 3f). The different rotations of the device for different steps during the POEM procedure can be appreciated in $>\mathbf{F i g} . \mathbf{2 a}$, > Fig. $\mathbf{2 b}$, and $>$ Fig. 2 c.

STER was performed using the same technique and settings on radiofrequency generator as POEM. The submucosal tunnel was extended for about $1 \mathrm{~cm}$ beyond the tumor, followed by 


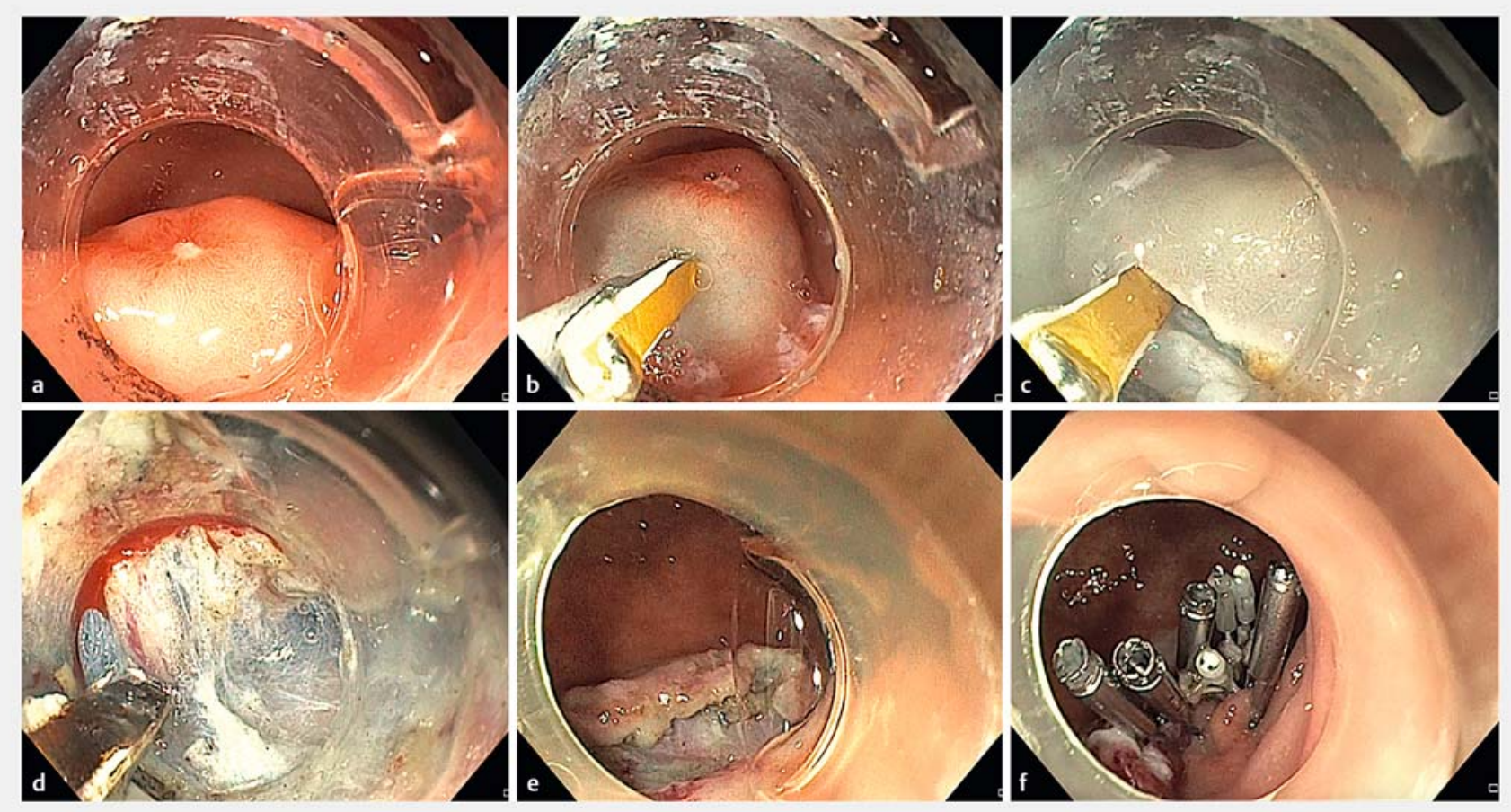

- Fig. 4 Endoscopic submucosal dissection of a gastric neuroendocrine tumor (NET). a Endoscopic view of gastric NET along greater curvature of stomach. $\mathbf{b}$ Submucosal lifting injection with the integrated needle system. c Mucosal incision. $\mathbf{d}$ Submucosal dissection below the tumor. e Submucosal defect after complete resection. $\mathbf{f}$ Closure of the defect using loop-ring technique.

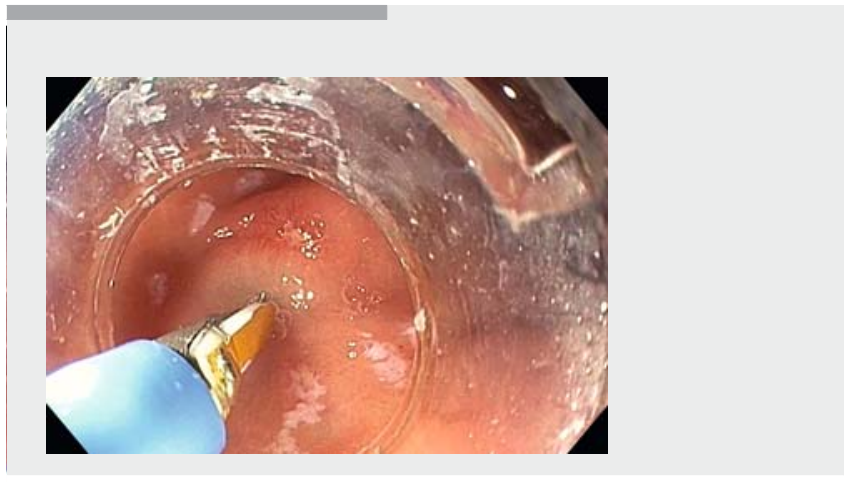

Video 1 ESD of gastric neuroendocrine tumor with bipolar device.

dissection of the tumor from the surrounding attachments. The tumor was finally removed using a standard polypectomy snare.

Steps in ESD included: circumferential marking of the lesion (gastric ESD), submucosal lifting injection ( $\mathbf{F i g . 4 a}$ and $\triangleright$ Fig. 4b), mucosal incision ( $\triangleright$ Fig. 4c), submucosal dissection ( $\triangleright$ Fig. $\mathbf{4 d}$ ), and retrieval of the specimen using a polypectomy snare. Closure of the post-ESD defect was left to the discretion of the endoscopist performing the procedure ( $\vee$ Fig. 4 e and $\triangleright \mathbf{F i g . 4 f}$ ). The settings on the radiofrequency generator used for ESD were similar to that used during POEM and STER ( $\vee$ Video 1 ).

\section{Definitions}

Completion of the entire procedure using the same device without the need for an alternative knife or coagulation forceps was considered as technical success.

AEs were defined as per the ASGE lexicon for endoscopic AEs. Mild, moderate, and severe AEs were classified according to the additional length of hospital stay i. e. $\leq 3$ nights (mild), 4 to 10 nights (moderate) and >10 nights (severe) [11]. Mucosal injuries during POEM or STER and inadvertent muscular injuries during ESD requiring closure with standard endoclips were considered as minor AEs. Insufflation related adverse events like capno-peritoneum and capno-thorax requiring intraprocedural needle drainage were also considered as mild [12].

\section{Statistics}

Data are presented as mean \pm SD or median (range). They were analyzed using MedCalc for Windows, version 12.2.1.0 (MedCalc Software, Ostend, Belgium).

\section{Results}

Ten patients (mean age $42.7 \pm 14.64$ years, 5 males) underwent $\operatorname{POEM}(n=7), \operatorname{ESD}(n=2)$ and STER $(n=1)$ using the new device. The subtypes of achalasia were type II (5) and type III (2). The patients with achalasia $(6,85.71 \%)$ were treatment-naïve. STER was performed for a subepithelial lesion arising from the second layer (muscularis propria) in the lower esophagus $(2.8 \times 2 \mathrm{~cm}$ on EUS $)$. Indications for ESD included gastric neu- 
- Table 1 Endoscopic submucosal dissection and tunneling procedures using novel bipolar radiofrequency device.

\begin{tabular}{|c|c|c|c|c|c|c|c|c|c|}
\hline Case & Sex & $\begin{array}{l}\text { Age, } \\
\text { years }\end{array}$ & Indication & $\begin{array}{l}\text { Prior inter- } \\
\text { vention }\end{array}$ & $\begin{array}{l}\text { Proce- } \\
\text { dure }\end{array}$ & $\begin{array}{l}\text { No. of } \\
\text { clips } \\
\text { used }\end{array}$ & $\begin{array}{l}\text { Procedure } \\
\text { duration } \\
\text { (minutes) }\end{array}$ & Adverse events & $\begin{array}{l}\text { Exchange of } \\
\text { accessories }\end{array}$ \\
\hline 1 & $\mathrm{~F}$ & 35 & Type II AC & LHM, PBD & POEM & 6 & 64 & Retroperitoneal $\mathrm{CO}_{2}$ & Yes $^{1}$ \\
\hline 2 & M & 71 & Esophageal SET & No & STER & 5 & 122 & No & No \\
\hline 3 & M & 52 & Type III AC & No & POEM & 4 & 61 & Capno-peritoneum & No \\
\hline 4 & $\mathrm{~F}$ & 24 & Type II AC & No & POEM & 5 & 28 & No & Yes $^{2}$ \\
\hline 5 & M & 30 & Type II AC & No & POEM & 7 & 52 & No & No \\
\hline 6 & $\mathrm{~F}$ & 45 & Type II AC & PBD & POEM & 5 & 25 & No & No \\
\hline 7 & $\mathrm{~F}$ & 26 & Type II AC & No & POEM & 6 & 43 & No & No \\
\hline 8 & $\mathrm{~F}$ & 46 & Type III AC & No & POEM & 4 & 78 & Empyema & No \\
\hline 9 & M & 42 & Gastric NET & Biopsy & ESD & 5 & 18 & No & No \\
\hline 10 & M & 56 & Rectal Polyp & EMR & ESD & 0 & 54 & No & No \\
\hline \multicolumn{10}{|c|}{$\begin{array}{l}\text { AC, achalasia cardia; LHM, laparoscopic Heller's myotomy; PBD, pneumatic balloon dilatation; POEM, per-oral endoscopic myotomy; TTJ, triangular tip knife J; SET, } \\
\text { sub-epithelial tumor; NET, neuroendocrine tumor; ESD, endoscopic submucosal dissection; EMR, endoscopic mucosal resection } \\
{ }^{1} \text { Exchanged with triangular knife for submucosal tunneling; } \\
{ }^{2} \text { Exchanged with coagulation forceps for hemostasis }\end{array}$} \\
\hline
\end{tabular}

roendocrine tumor $(\mathrm{NET}, 1.5 \times 1 \mathrm{~cm})$ and one laterally spreading rectal polyp $(3 \mathrm{~cm})(\triangleright$ Table $\mathbf{1})$.

\section{Procedure details and outcomes}

Procedures were successfully accomplished in all the cases. POEM was performed via the posterior route in all cases. Mean time taken for tunneling procedures (POEM and STER) was $59.12 \pm 31.12$ minutes. Mean duration of POEM procedure was significantly higher in cases with type III achalasia and prior Heller's myotomy as compared to other cases with achalasia $(67.67 \pm 9.07$ vs $37 \pm 12.73 ; P=0.017)$. Procedure durations for rectal and gastric ESD were 54 and 18 minutes, respectively.

In eight patients ( $80 \%$ ), the entire procedure could be completed using the new device. In addition to the novel device, accessories including a triangular tip knife and coagulation forceps were used in two cases (Case 1 and Case 4) for submucosal dissection and hemostasis, respectively. In the first case, submucosal fibrosis was evident near the gastroesophageal junction due to previous pneumatic dilatation and Heller's myotomy. The triangular knife was used to complete the distal part of submucosal tunneling in this case. In Case 4, hemostasis could not be achieved with the new device due to imperfect apposition of the device with the bleeding point. In other cases, the procedures were accomplished without the need to exchange accessories ( $\triangleright$ Table $\mathbf{1}$ ).

Clinical success with resolution of dysphagia (Eckardt $\leq 3$ ) was noted in all patients who underwent POEM. En-bloc resection was feasible in patients who underwent STER and ESD.

\section{Adverse events}

There were no major AEs. There was one moderate AE, left empyema requiring drainage in a patient who underwent POEM. Minor AEs included capno-peritoneum $(n=1)$ requiring needle drainage and retroperitoneal $\mathrm{CO}_{2}(n=1)$ accumulation demanding temporary cessation of the procedure. There were no cases with inadvertent injury to the mucosa or muscle.

\section{Discussion}

In this case series, we demonstrated use of a novel bipolar device for submucosal tunneling procedures and ESD.

Submucosal tunneling procedures and ESD are widely performed for a variety of indications in the gastrointestinal tract. These are complex therapeutic endoscopic procedures and carry a risk of collateral damage to the muscle or mucosa, especially when performed during the learning curve [12]. The technical difficulty associated with these procedures can be reduced with experience as well as through improvement in the currently available electrosurgical knives $[4,7,13]$.

In this study, we evaluated the utility of a new bipolar device for various procedures involving submucosal tissue dissection including POEM, ESD, and STER. This novel device is equipped with an injection needle and uses radiofrequency energy for cutting and microwave energy for coagulation [14]. This implies that all the steps in tunneling and ESD procedures, including submucosal injection, dissection, and hemostasis can be accomplished with a single device without the need for exchange of accessories. Our group has previously reported on use of this device in two cases (one POEM and one STER) $[9,10]$. In the current study, we further evaluated the utility of this knife for POEM, STER, and ESD. The majority of the procedures could be accomplished successfully using the same device. Alternate accessories were used for dissection and coagulation in Case 1 and Case 4, respectively. The main reason for the exchange of accessories was the relative unfamiliarity of the endoscopist as well as the assistant with the new device in the initial cases. In 
addition, coordination is required between the endoscopist and the assistant for orienting the device for dissection, myotomy, and coagulation. With improvement in the experience of the endoscopist as well as better coordination with the assistant, there was no requirement for exchange of accessories in the subsequent six cases.

Mean procedure time was relatively high $(59.12 \pm 31.12 \mathrm{~min}$ utes) in the current series. It can be argued that the time required to accomplish the procedures should have been less while using this multipurpose device. Inclusion of relatively difficult presentations such as type III achalasia requiring a long myotomy (two cases), and patients with a history of prior treatment failure (EMR 1, Heller's myotomy 1 ) probably lead to an increased overall procedure duration.

We also evaluated the safety of procedures while using this device. There were no severe AEs in the current series. There were no inadvertent thermal injuries to the mucosa or muscle and the procedures could be completed with minimal charring of the tissues. The insulated surface at the top and protective hull at the bottom help prevent collateral damage to the mucosa and muscle fibers during submucosal tunneling procedures and ESD, respectively.

The implications of our series are as follows. Use of this multi-purpose device is likely to reduce the requirement for exchanging accessories. This, in turn, would reduce the technical difficulty and operating duration of these procedures. In addition, the bipolar nature of the device and its protective upper and lower surfaces minimize the chances of inadvertent injury to mucosa and muscle fibers.

While use of THE new device was largely safe and effective in the current series, a few points are worth mentioning. First, use of this device requires a therapeutic endoscope with a large-diameter channel, which may compromise the flexibility offered by slimmer endoscopes to some extent. For the same reason, some endoscopists do not prefer a therapeutic channel endoscope for performing ESD or POEM procedures. Second, a separate electrosurgical generator is required while using this device. This adds to the overall cost of the procedure. However, the reduced requirement for accessories such as an injection needle and coagulation forceps may compensate for the extra cost. Third, close coordination is required between the endoscopist and the assistant for desired rotation of the device for optimal cutting. Fourth, we did not include sigmoid esophagus and non-achalasia esophageal motility disorders due to presumed difficulty with using a new device for these procedures. Finally, most procedures in this series were POEM for achalasia. ESD and STER were performed in fewer cases. Therefore, the feasibility and safety of the novel bipolar device for these procedures should be explored further in studies with larger sample size.

\section{Conclusion}

Submucosal dissection using a new bipolar device is feasible and safe. The reduced requirement of exchange of accessories and insulated surface may increase the technical ease and safety of complex endoscopic procedures. Comparative trials with a large sample size are required to establish the role of this novel device for performing endoscopic dissection procedures.

\section{Competing interests}

Dr. Tsiamoulos is a consultant agreement for Creo Medical and has received educational grants from Norgine and loan equipment from Olympus and Pentax.

\section{References}

[1] Nabi Z, Nageshwar Reddy D et al. Recent Advances in Third-Space Endoscopy. Gastroenterol Hepatol (NY) 2018; 14: 224-232

[2] Kumta NA, Kedia P, Lambroza A et al. Successful peroral endoscopic myotomy with use of a single instrument multipurpose knife. Gastrointest Endosc 2014; 80: 1168

[3] Bittinger M, Messmann $\mathrm{H}$. Use of the stag-beetle knife for peroral endoscopic myotomy for achalasia: a novel method for myotomy. Gastrointest Endosc 2015; 82: 401-402

[4] Tanaka S, Toyonaga T, Kawara F et al. Peroral endoscopic myotomy using FlushKnife BT: a single-center series. Endosc Int Open 2017; 5: E663-E669

[5] Shimizu T, Fortinsky KJ, Chang KJ. Early experience with use of an endoscopic "hot" scissor-type knife for myotomy during per-oral endoscopic myotomy procedure. VideogastrointestinalE 2019; 4: 182-184

[6] Cai MY, Zhou PH, Yao LQ et al. Peroral endoscopic myotomy for idiopathic achalasia: randomized comparison of water-jet assisted versus conventional dissection technique. Surg Endosc 2014; 28: 11581165

[7] Nabi Z, Ramchandani M, Chavan R et al. Outcome of peroral endoscopic myotomy in achalasia cardia: Experience with a new triangular knife. Saudi J Gastroenterol 2018; 24: 18-24

[8] Nabi Z, Ramchandani M, Jagtap $\mathrm{N}$ et al. Endoscopic treatment of Zenker's diverticulum using a new triangle tip knife. Ann Gastroenterol 2019; 32: 650-653

[9] Nabi Z, Ramchandani M, Chavan R et al. Endoscopic dissection of an esophageal submucosal tumor using a novel bipolar radiofrequency device. Endoscopy 2020; 52: E257-E258

[10] Nabi Z, Ramchandani M, Darisetty S et al. Peroral endoscopic myotomy in a patient with failed Heller's myotomy by use of a novel bipolar radiofrequency device. VideogastrointestinalE 2020: doi:10.1016/j. vgie.2019.12.009

[11] Cotton PB, Eisen GM, Aabakken L et al. A lexicon for endoscopic adverse events: report of an ASGE workshop. Gastrointest Endosc 2010; 71: 446-454

[12] Nabi Z, Reddy DN, Ramchandani M. Adverse events during and after per-oral endoscopic myotomy: prevention, diagnosis, and management. Gastrointest Endosc 2018; 87: 4-17

[13] Ohara Y, Toyonaga T, Hoshi N et al. Usefulness of a novel slim type FlushKnife-BT over conventional FlushKnife-BT in esophageal endoscopic submucosal dissection. World J Gastroenterol 2017; 23: 16571665

[14] Tsiamoulos ZP, Sebastian J, Bagla N et al. A new approach to endoscopic submucosal tunneling dissection: the "Speedboat-RS2" device. Endoscopy 2019; 51: E185-E186 